\title{
HSC/98/01
}

\section{Origins of the scaling behaviour in the dynamics of financial data}

\author{
Aleksander Weron* \\ Szymon Mercik** \\ Rafał Weron***
}

* Hugo Steinhaus Center, Wrocław University of Technology, Poland

** Institute of Physics, Wrocław University of Technology, Poland

*** Institute of Mathematics, Wrocław University of Technology, Poland

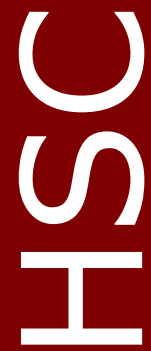

Hugo Steinhaus Center Wrocław University of Technology Wyb. Wyspiańskiego 27, 50-370 Wrocław, Poland http://www.im.pwr.wroc.pl/ hugo/ 


\title{
Origins of the scaling behaviour in the dynamics of financial data
}

\author{
Aleksander Weron ${ }^{a}$, Szymon $_{\text {Mercik }}^{b}$, Rafal Weron ${ }^{c, 1}$ \\ ${ }^{a}$ Hugo Steinhaus Center for Stochastic Methods, \\ ${ }^{b}$ Institute of Physics, \\ ${ }^{c}$ Institute of Mathematics, \\ Wroclaw University of Technology, 50-370 Wroclaw, Poland
}

\begin{abstract}
:
The Conditionally Exponential Decay (CED) model is used to explain the scaling laws observed in financial data. This approach enables us to identify the distributions of currency exchange rate or economic indices returns (changes) corresponding to the empirical scaling laws. This is illustrated for daily returns of the Dow Jones Industrial Average (DJIA) and the Standard \& Poor's 500 (S\&P500) indices as well as for high frequency returns of the USD/DEM exchange rate.
\end{abstract}

PACS: 02.50.-r; 87.10.+e

Keywords: Econophysics, scaling law, CED model, high frequency data

\section{Introduction}

As has been reported recently, many complex systems in economy display scaling properties $[1,2,3,4,5,6,7]$ similar to those found in the social [8], physical [9, 10], and biological sciences [11]. However, in contrast to the existing literature, here we also show how to identify the distributions of returns corresponding to the empirical scaling laws. For this purpose, we adopt the Conditionally Exponential Decay (CED) model [12], which has been developed for complex systems. It uniquely leads to two power-laws describing asset returns $[13,14]$ and immediately explains the observed scaling laws. The presented approach should provide a framework for proper understanding of scaling behaviour not only in finance but also in other sciences.

It is well known that market returns are not normally distributed $[15,16,17]$. A variety of alternatives to the normal law can be found in literature and it is undeniable that as long as the distribution that is implied by these models is more

\footnotetext{
${ }^{1}$ Corresponding author. E-mail: rweron@im.pwr.wroc.pl
} 
leptokurtic than the Gaussian law, it will provide a better fit. However, some alternatives to the normal distribution were often rejected even though they fit the data without modification. Why? - Standard statistical analysis can not be applied using those distributions. Besides that, it is one thing to fit given data well through the choice of a "good distribution", but it is an entirely different matter to explain return's data through the use of a statistical model that predicts the data's main characteristics. To deal with such a problem, i.e. to describe the global distributional structure of asset returns, we employ here the Conditionally

Exponential Decay model $[12,13,14]$, which is based on asymptotic behaviour of complex stochastic systems and current developments of chaos theory.

The fractal view starts from a basic principle: analyze an object on different scales, with different degrees of resolution, then compare and interrelate the results. For financial time series, this means using different time yardsticks $(\Delta t)$ from hourly through daily to monthly and yearly, within the same study. This is far from the conventional time series analysis, which focuses on regularly spaced observations with fixed time intervals. In place of the traditional Efficient Market Hypothesis, the Fractal and the Heterogeneous Market Hypotheses have been proposed $[1,17,18,19,20]$. They emphasize the impact of information and investment horizons on the behaviour of investors. The basic assumptions of these hypotheses are the following [19]: the market is made up of many individuals with a large number of different investment horizons and is heterogeneous in the geographic location of the participants; information has a different impact on different investment horizons; prices reflect a combination of short-term technical trading and long-term fundamental valuation.

\section{The CED model}

The CED model clarifies the ideas of the Fractal and the Heterogeneous Market Hypotheses and provides a rigorous mathematical framework for further analysis of financial complex processes. In this model $[13,14,17]$ each $i$ th investor is related to a cluster of agents acting simultaneously on common markets. The influence of this cluster of agents is of the type of short-range interactions and is reflected by a random risk-aversion factor $A_{i}$. Interactions of the long-range type are imposed on the $i$ th investor by the inter-cluster relationship manifested by random risk factors $B_{j}^{i}$ for all $j \neq i$. They reflect how fast the information flows to the $i$ th investor. These assumptions lead to a two power-law for complex financial systems described by the following CED probability density of returns

$$
f(r)= \begin{cases}C_{1}(\lambda r)^{\alpha-1} & \text { for } 0 \leq \lambda r \ll 1, \\ C_{2}(\lambda r)^{-\frac{\alpha}{k}-1} & \text { for } \lambda r \gg 1,\end{cases}
$$

where $C_{1}=\alpha \lambda$ and $C_{2}$ is a function of all three CED parameters: $\alpha$ - the shape, $\lambda$ - the scale, and $k$. The last parameter decides how fast the information flow is spread out in the market. 

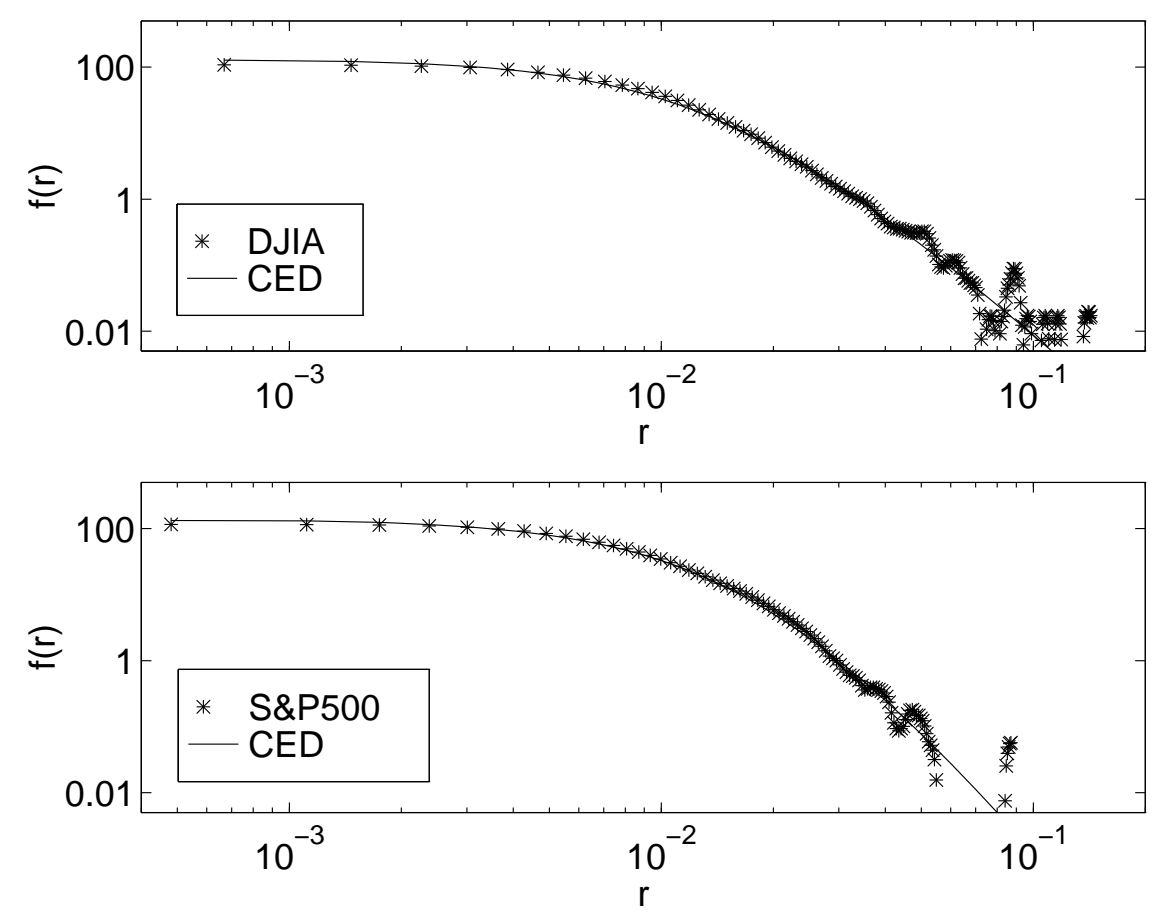

Figure 1: Log-log plots (double logarithmic plots) of the empirical densities (kernel estimators) and the approximating CED densities for positive daily returns of the DJIA index (top) and the S\&P500 index (bottom).

Consider now two data sets consisting of daily returns of the DJIA and the S\&P500 indices. For each set of data we run maximum likelihood estimation of the parameters $\alpha, \lambda$ and $k$, both for positive and for absolute value of negative returns.

The first data set contained observations of the DJIA index during the period from January 2, 1901 to May 17, 1996. The 26226 daily returns of the DJIA index were split to positive and negative returns, and the analysis was carried out for both (recall that if $s_{t}$ is the daily observation of day $t$ for the DJIA index, then $r_{t}=\log s_{t}-\log s_{t-1}$ is the daily return of day $t$ ). The minimization procedure yielded the following parameters: $\alpha^{+}=1.089, \lambda^{+}=156.36, k^{+}=0.2557$ and $\alpha^{-}=1.1097, \lambda^{-}=142.90, k^{-}=0.2889$ for positive and absolute value of negative returns, respectively. A log-log plot of the empirical density and the approximating CED density for positive returns is shown in Fig.1.

The second data set contained observations of the S\&P500 index during the period from July 2, 1962 to December 31, 1991. The 7420 daily returns of the S\&P500 index were split to positive and negative returns, and the analysis was carried out for both. The minimization procedure yielded the following parameters: $\alpha^{+}=1.0978, \lambda^{+}=164.48, k^{+}=0.1781$ and $\alpha^{-}=1.0988, \lambda^{-}=156.10, k^{-}=0.1548$ for positive and absolute value of negative returns, respectively. A log-log plot of the empirical density and the approximating CED density for positive returns is shown in Fig.1. 


\section{The scaling of the probability distribution of returns}

Now, after a brief introduction to the CED model, let us turn to the scaling behaviour in the dynamics of financial data. In a series of publications $[2,4,5]$ the authors reported that the scaling of the probability distribution of the S\&P500 index returns can be described by a non-Gaussian process with dynamics that, for the central part of the distribution, correspond to that predicted for Lévy stable symmetric process [21] with the index parameter $\alpha=1.40$. A relation of this scaling law to the well known scaling law for volatility $[1,18,19]$ has been a challenging problem $[6,19]$. In this paper we show that both empirical scaling laws can be deduced from the universal behaviour of a complex system described by the CED model.

But before we do that observe that stable distributions do not play any special role, since the scaling of the probability distribution for the central part - more precisely at point zero for symmetric distributions - holds in general for many distributions which do not have to belong to the class of Lévy stable laws. Indeed, if $f_{\lambda}(x)$ denotes the empirical density with a unique scale parameter $\lambda$, i.e. $f_{\lambda}(x)=\lambda f_{1}(\lambda x)$, which satisfies the relation (in time)

$$
\lambda \equiv \lambda(\Delta t)=C \cdot(\Delta t)^{D}
$$

then the density has the following scaling power-law

$$
\log \left(f_{\lambda(\Delta t)}(0)\right)=\log \left(f_{1}(0)\right)+\log C+D \cdot \log (\Delta t) .
$$

This is illustrated in Fig.2 for three different distributions, including the Lévy stable law, approximating the returns of the USD/DEM exchange rate (the data set is described in the next section). In contrast to the earlier work [2], we have found that the returns are not symmetric and that the index parameter $\alpha$ of the Lévy stable law is increasing with $\Delta t$ from about 1.5 to 1.8 . What's more, as a special case - taking $f$ as a symmetric Lévy stable density with index $\alpha$ and scale factor $\gamma$ - one can easily get the scaling result [2] by letting $C=\gamma^{-\alpha}$ and $D=-1 / \alpha$. However, let us underline here that it is not possible to derive from formula (3) the important scaling law for volatility discovered in financial markets $[1,18,19]$.

\section{The scaling law for volatility}

In the last section of this paper we discuss the scaling law for volatility using high frequency data from the foreign exchange (FX) market. Namely, the data set considered is the USD/DEM exchange rate for the whole 1996 year, released by Olsen \& Associates for the Second International Conference on High Frequency Data in Finance, Zurich, April 1-3, 1998.

Adequate analysis of the high frequency (intra-daily) data relies on an explicit definition of the variables under study. These include the price, the change of price, and the volatility [19]. The price at time $t_{i}$ is defined as $x\left(t_{i}\right) \equiv \frac{1}{2}\left[\log p_{b i d}\left(t_{i}\right)+\right.$ 


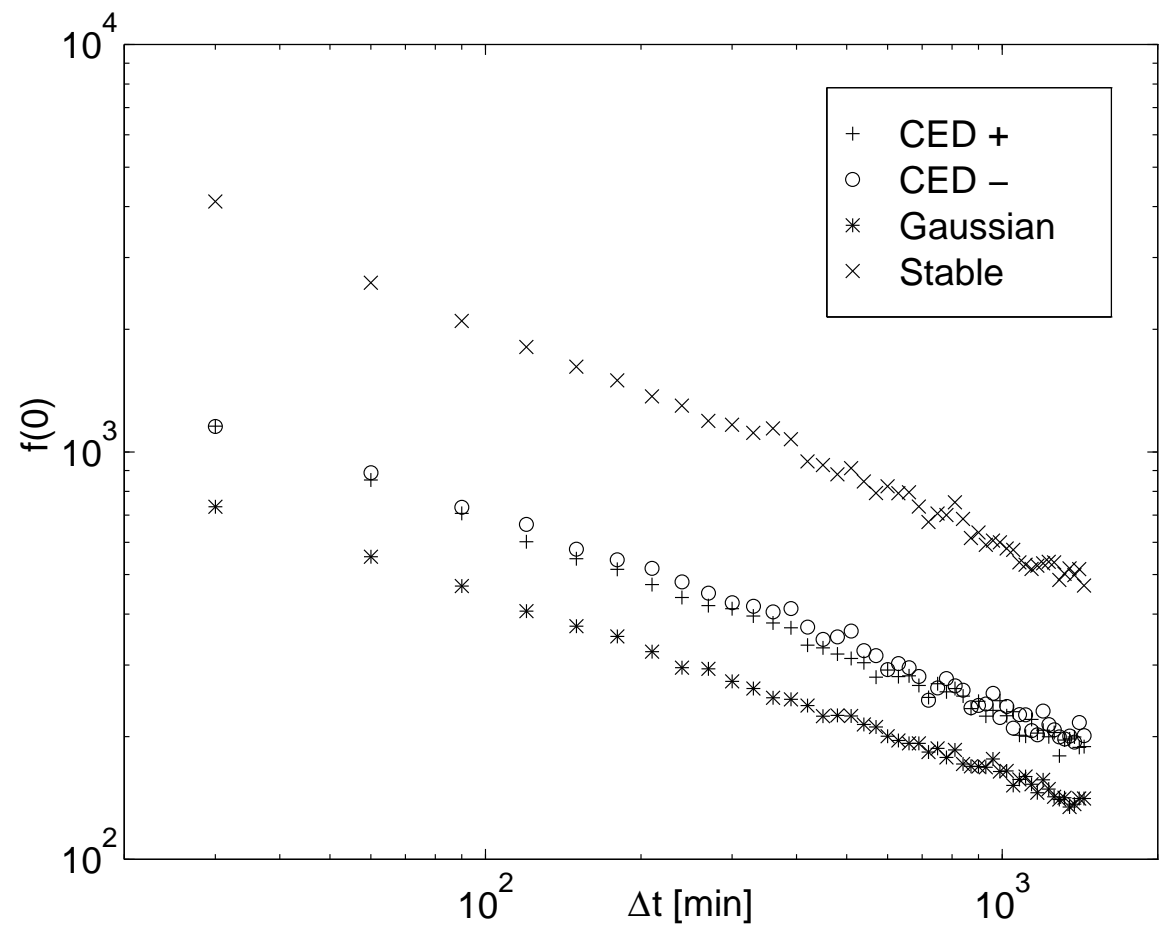

Figure 2: Log-log plot of the densities approximating the USD/DEM exchange rate returns at point zero as a function of the time sampling interval $\Delta t$ for three different distributions: CED (separately for positive and absolute value of negative returns), Gaussian, and Lévy stable. For better presentation only the last two terms of the right hand side of formula (3) are taken into account. A scaling power law behaviour is observed for time intervals spanning two orders of magnitude. The slopes of the least squares regression for these distributions are: $D_{C E D+}=-0.4743$, $D_{C E D-}=-0.4811, D_{\text {Gauss }}=-0.4377$, and $D_{\text {stable }}=-0.5436$.

$\left.\log p_{a s k}\left(t_{i}\right)\right]$, where $\left\{t_{i}\right\}$ is a sequence of regular spaced (30 minutes for this data set) in time data and $p_{b i d}\left(t_{i}\right)\left(p_{a s k}\left(t_{i}\right)\right)$ is the arithmetic average of the bid (ask) quotes just prior to and just after time $t_{i}$. The definition takes the average of the bid and ask price rather than either the bid or the ask series as a better approximation of the transaction price. The change of price $r\left(t_{i}\right)$ at time $t_{i}$ is defined as $r\left(t_{i}, \Delta t\right) \equiv$ $\left[x\left(t_{i}\right)-x\left(t_{i}-\Delta t\right)\right]$, where $\Delta t$ is the time interval $(\Delta t=30$ minutes, $\Delta t=1$ hour, etc.). In fact, this is the change of the logarithmic price and is often referred to as return. The volatility $v\left(t_{i}\right)$ at time $t_{i}$ is defined as

$$
v\left(t_{i}\right) \equiv \frac{1}{n} \sum_{k=1}^{n}\left|r\left(t_{i-k}, \Delta t\right)\right| .
$$

To illustrate the dependence of the empirical result from the time horizons $(\Delta t)$ we considered 48 types of returns: $30 \mathrm{~min}$., $60 \mathrm{~min} .$, ..., $1440 \mathrm{~min}$. (= 24 hours). For example, for positive returns and $\Delta t=30$ minutes (see Fig.3), the minimization procedure yielded the following parameters: $\alpha^{+}=1.0781, \lambda^{+}=1156.3, k^{+}=$ 

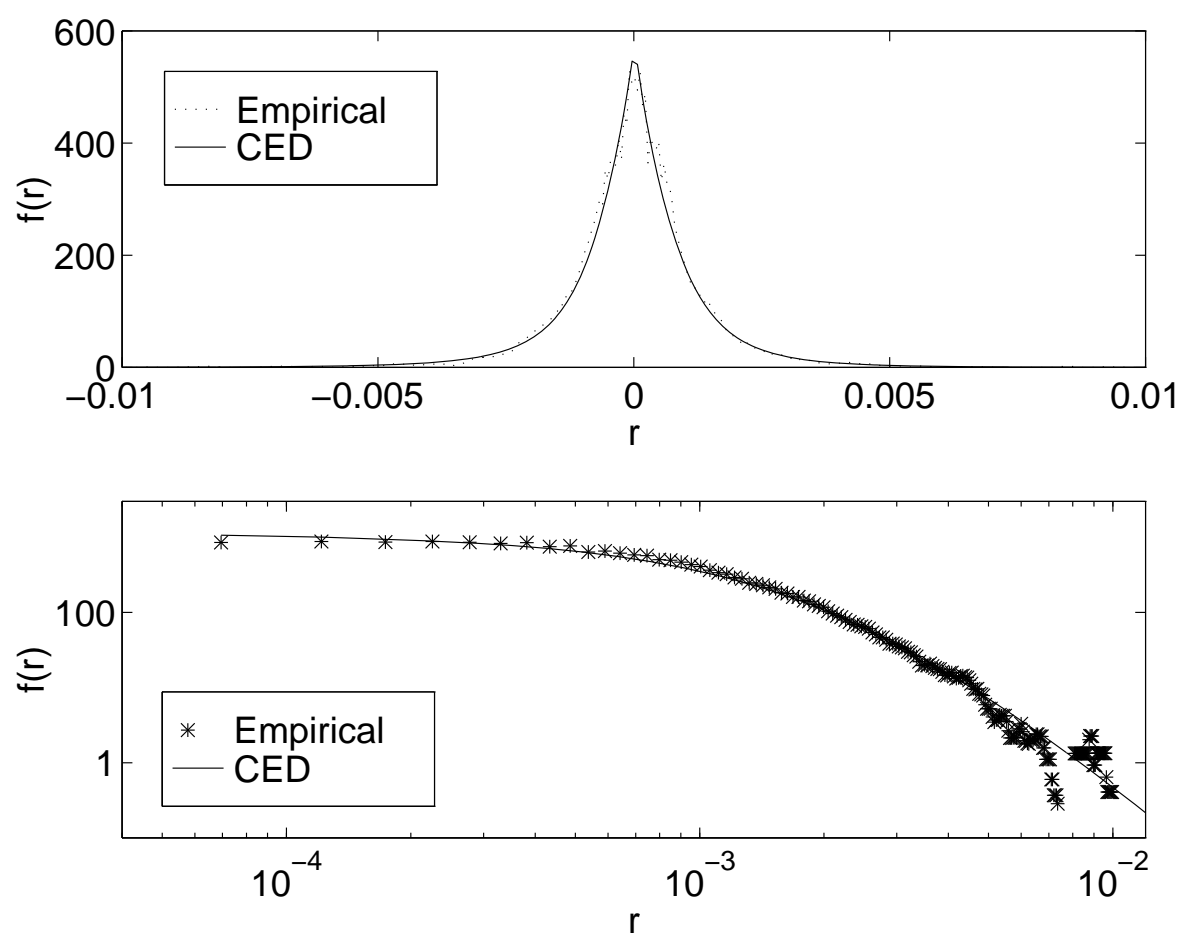

Figure 3: Plot of the empirical density for returns of the USD/DEM exchange rate and the approximating CED density for $\Delta t=30$ minutes $(t o p)$. Log-log plot of the above densities for positive returns only (bottom).

0.2508 .

First observe that from the general scaling property (2) for the density (1) of a CED model with parameter $\alpha=1$ and $\lambda r \ll 1$ we have $f_{\lambda}(r)=\lambda(\lambda r)^{0}=\lambda$. In particular, for $r=0$ we immediately get $f_{\lambda}(0)=\lambda$. Hence the scaling property $(3)$ takes the form $\log \left(f_{\lambda(\Delta t)}(0)\right)=\log C+D \cdot \log (\Delta t)$. Moreover, evaluating the mean value of the CED distribution and using the following two facts - formula (4) gives a good estimator of the mean value of the probability distribution of returns; asset returns are well described by the CED distribution - we get the empirical scaling law for volatility

$$
v(\Delta t)=C \cdot(\Delta t)^{H} .
$$

The comparison of the scaling laws for empirical data (the USD/DEM exchange rate) and for the estimated CED model is illustrated in Fig.4. Notice that the fit is extremely good and that it is much better than in [14] (see Fig.3), where only an approximation of the mean value of the CED distribution by $1 / \lambda$ was used.

A log-log plot of the estimating CED densities for $\Delta t$ 's ranging from 30 to 1440 minutes $(=24$ hours) is shown in Fig.5. Observe essential differences in the shapes of these densities supporting the hypothesis of a scaling law as a function of the time interval ranging from a few minutes up to a day. 


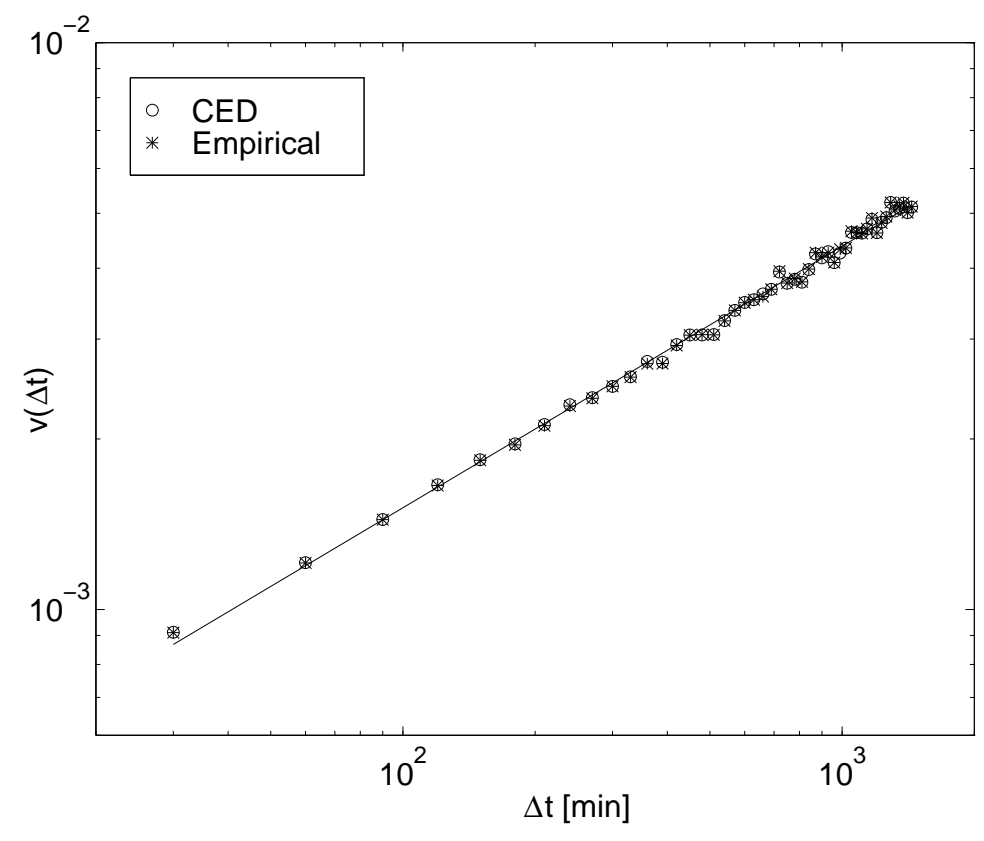

Figure 4: Scaling laws for volatility of the USD/DEM exchange rate and the approximating CED densities (each point represents an average of a CED density for positive and absolute value of negative returns) for all $\Delta t$ 's. The solid line is the least squares regression line for the CED densities with slope $H_{C E D}=0.4595$. For comparison, the slope for the empirical data is $H_{e m p}=0.4614$.

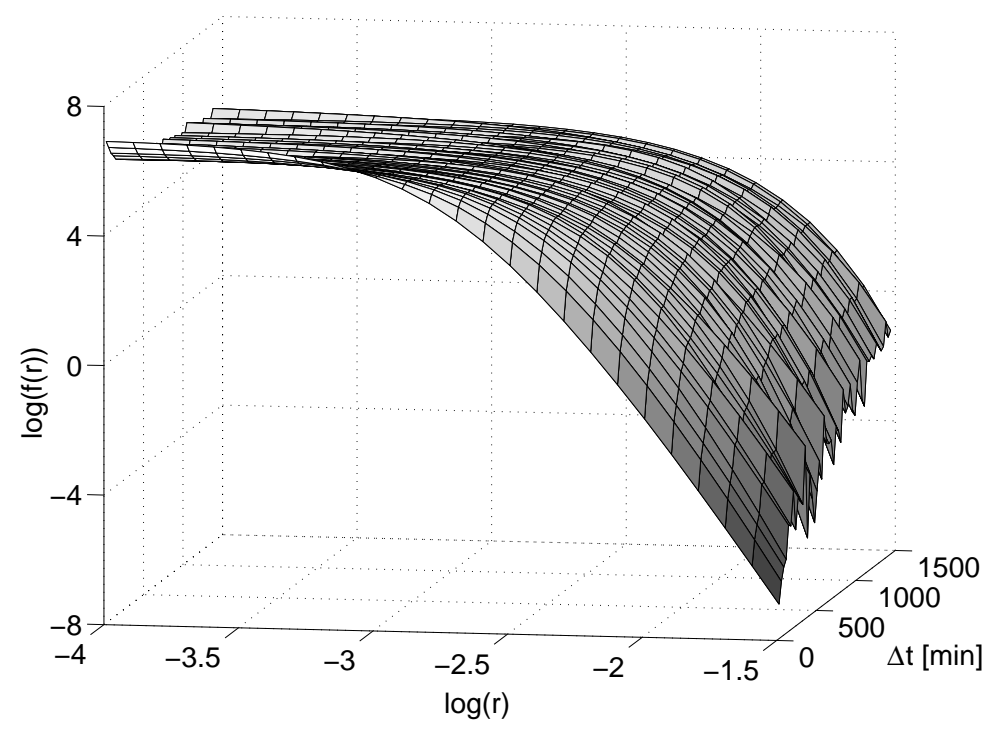

Figure 5: 3D plot (on a double logarithmic scale) of the estimating CED densities for $\Delta t$ 's ranging from 30 to 1440 minutes (= 24 hours). Only positive returns are displayed. 


\section{References}

[1] U.A. Müller, M.M. Dacorogna, R.B. Olsen, O.V. Pictet, M. Schwarz, C. Morgenegg, J. Banking \& Finance 14 (1990) 1189.

[2] R.N. Mantegna, H.E. Stanley, Nature 376 (1995) 46.

[3] M.H.R. Stanley, L.A.N. Amaral, S.V. Buldyrev, S. Havlin, H. Leschhorn, P. Maass, M.A. Salinger, H.E. Stanley, Nature 379 (1996) 804.

[4] Y. Liu, P. Cizeau, M. Meyer, C.-K. Peng, H.E. Stanley, Physica A 245 (1997) 437.

[5] P. Cizeau, Y. Liu, M. Meyer, C.-K. Peng, H.E. Stanley, Physica A 245 (1997) 441.

[6] S. Galluccio, G. Caldarelli, M. Marsili, Y.-C. Zhang, Physica A 245 (1997) 423.

[7] P. Gopikrishnan, M. Meyer, L.A.N. Amaral, H.E. Stanley, Euro. Phys. J. B 3 (1998) 139.

[8] G.K. Zipf, Human Behavior and the Principle of Least Effort, Addison-Wesley, Reading MA, 1949.

[9] K. Weron, A. Jurlewicz, J. Phys. A: Math. Gen. 26 (1993) 395.

[10] A.K. Jonscher, Universal Relaxation Law, Chelsea Dielectrics, London, 1996.

[11] I. Peterson, The Jungles of Randomness, Viking, New York, 1997.

[12] A. Jurlewicz, A. Weron, K. Weron, Appl. Math. 23 (1996) 379.

[13] S.T. Rachev, A. Weron, K. Weron, Appl. Math. Lett. 10 (1997) 5.

[14] R. Weron, K. Weron, A. Weron, Physica A (1998) to appear.

[15] B.B. Mandelbrot, J. Business 36 (1963) 394.

[16] E. Fama, J. Business 38 (1965) 34.

[17] A. Weron, R. Weron, Financial Engineering: Derivatives Pricing, Computer Simulations, Market Statistics, (in Polish), WNT, Warsaw, 1998.

[18] M.M. Dacorogna, U.A. Müller, R.J. Nagler, R.B. Olsen, O.V. Pictet, J. Intern. Money Finance 12 (1993) 413.

[19] D.M. Guillaume, M.M. Dacorogna, R.R. Dave, U.A. Müller, R.B. Olsen, O.V. Pictet, Finance Stochast. 1 (1997) 95.

[20] E.E. Peters, Fractal Market Analysis: Applying Chaos Theory to Investment and Economics, Wiley \& Sons, New York, 1994.

[21] A. Janicki, A. Weron, Simulation and Chaotic Behavior of $\alpha$-Stable Stochastic Processes, Marcel Dekker, New York, 1994. 


\section{HSC Research Report Series 1998}

For a complete list please visit http://ideas.repec.org/s/wuu/wpaper.html

01 Origins of the scaling behaviour in the dynamics of financial data by Aleksander Weron, Szymon Mercik and Rafał Weron 